\title{
A heat and mass transfer study of carbon paste baking
}

\author{
Lindon Roberts $^{a}$, Ellen Nordgård-Hansen ${ }^{b}, \emptyset_{\text {yvind Mikkelsen }}{ }$, Svenn Anton Halvorsen $^{b}$, and Robert A. Van Gorder ${ }^{a *}$ \\ ${ }^{a}$ Mathematical Institute, University of Oxford, Andrew Wiles Building, Radcliffe Observatory Quarter, Woodstock Road, Oxford OX2 6GG United Kingdom \\ ${ }^{b}$ Teknova AS, Tordenskjolds gate 9, 5th floor, NO-4612 Kristiansand S, Norway \\ ${ }^{c}$ Elkem Carbon AS, P.O. Box 8040, Vaagsbygd, NO-4675 Kristiansand S, Norway \\ *Robert.VanGorder@maths.ox.ac.uk
}

\begin{abstract}
Ramming paste is a particular type of carbon paste which is used as lining for electric smelting furnaces and Hall-Héroult cells for the production of aluminium. The purpose of this lining is to form an impenetrable barrier, keeping the liquid within the furnace. If the lining has cracks or holes, then liquid can escape, which can lead to safety risks and financial losses, so the integrity of the lining is of great importance. In the present study, we develop a first principles mathematical model for the heat and mass transfer processes occurring during the baking of carbon paste. We then obtain numerical simulations using this model and compare the simulation results to experimental data, demonstrating that the model solutions do indeed describe and predict realistic behaviors of the carbon paste baking process. The simulations indicate a strong pressure buildup during the evaporation of water from fresh paste during the baking process, which is likely to lead to cracking of the paste as it hardens. Furthermore, we are able to show that more gradual heating during the baking process can lower the maximal pressures predicted by the model, which in turn may reduce the prevalence of cracks within the hardened paste.
\end{abstract}

Keywords: heat and mass transfer model, baking process, carbon paste, baking profile, thermal expansion

\section{Introduction}

Baking and similar processes arise in diverse industrial applications, such as metallurgy, structural engineering [1], soil mechanics [2], and food manufacturing [3]. These processes may include a number of physical phenomena, including: heat convection and conduction, driven primarily by an external heat supply; chemical reactions including phase changes; mass transport through porous media; and deformation and hardening of porous media. In the present paper, we shall be interested in the baking of carbon paste.

Carbon pastes have several uses in industrial metallurgy, such as self baking paste in Søderberg- and composite electrodes. Ramming paste is a particular type of carbon paste which is used as lining for electric smelting furnaces and Hall-Héroult cells for the production of aluminium. The purpose of the lining is to form an impenetrable barrier, keeping the liquid within the furnace. If the lining has cracks or holes, then liquid can escape, which can lead to safety risks and financial losses. Lining made from poor quality ramming paste can mean a shorter lifespan before replacement, which is a costly process due to the expense of shutting down a furnace, the re-lining of the furnace, and lost production. The lifespan of ramming paste may vary from 5-10 years for high quality paste to less than 1 year for low quality paste. A key part of the installation of ramming paste as a furnace lining is baking, where the paste is heated, causing it to harden and become impermeable. Samples of ramming paste which have cracked during the baking process are shown in Figure 1.

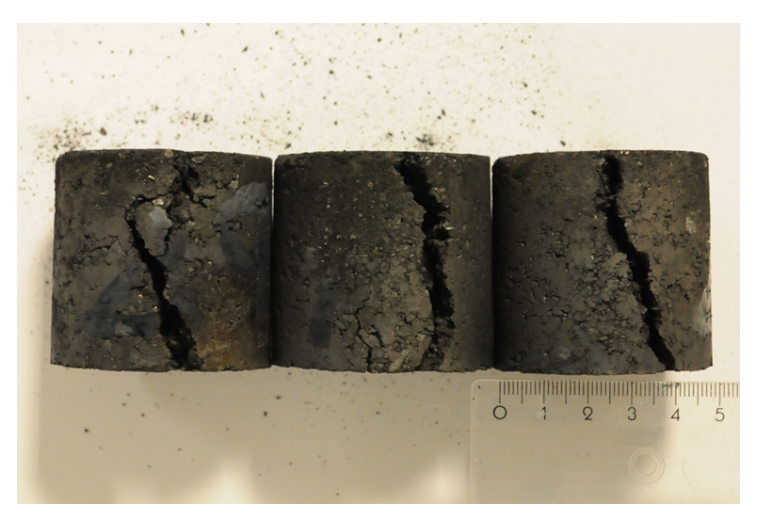

Figure 1: Samples of ramming paste which have cracked during baking. Source: Elkem Carbon.

Traditional ramming pastes are mostly made of granular anthracite (a type of coal, with similar properties to graphite), held together with a binder made from coal tar pitch. Coal tar pitch is a substance containing polycyclic aromatic hydrocarbons (PAHs), which are carcinogens. Exposure to coal tar pitch has been linked with increased risk of cancer in the skin, lungs, bladder, kidney, and other organs [4]. The installation of ramming paste within a furnace can expose workers to PAHs, even with safety precautions in place. To this end, Elkem, a large Norwegian producer of basic materials, has been developing non-toxic (so-called 'green') binders for ramming paste made of organic compounds.

Given the harsh environments in which the baking of ram- 

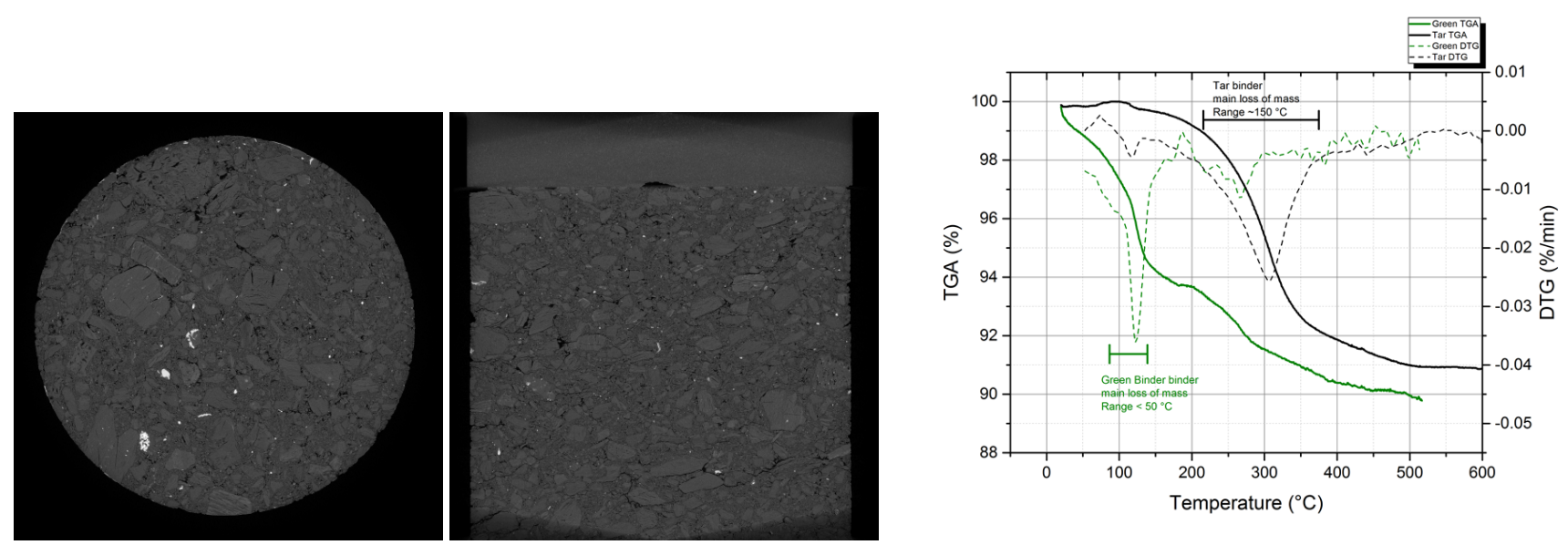

Figure 2: (left and middle): CT scans of baked ramming paste, using the new organic binder. Source: Elkem Carbon. (right) Mass loss profiles for coal tar pitch (black line) and green binders. The dashed lines represent the rates of change of mass. Source: Elkem Carbon.

ming paste occurs and the complexity of the processes involved, direct measurements can be difficult to obtain. In this situation, it is useful to have a mathematical model of the key physical processes, allowing for computational testing and predictions. This motivates us to develop such a model.

This paper is organised as follows. In Section 2, we outline the experimental setup we are modelling and then derive the mathematical model. This model is non-dimensionalised and simplified, providing insight into which physical processes are most important. Numerical simulations are presented in Section 3 , along with a discussion of fit to experimental data, qualitative model behaviour, and parameter sensitivity. Lastly, Section 4 provides a summary of the main results, as well as a list of suggestions for future work and improvements to the model.

\section{Mathematical model of carbon paste baking}

The process for the installation of ramming paste is: (i) line the furnace with paste, manually ramming (compressing) it into the correct shape; (ii) slowly heat the furnace to bake the paste; and (iii) lastly, as the paste heats, it solidifies to form an impenetrable layer (with the correct thermal and electrical properties). Typically, ramming paste consists of $80-85 \%$ granular anthracite (by mass) and 15-20\% coal tar pitch. In Elkem's new ramming paste, the composition is approximately $85 \%$ granular anthracite, $5 \%$ free water and $10 \%$ organic compounds (which are dissolved in the water). CT scans of the new paste are shown in Figure 2 - the large light grey pieces are anthracite, the dark grey areas are binder, and the black areas are voids.

Elkem has observed that paste using these new green binders exhibit a fast rate of mass loss during the baking process. This is shown in Figure 2, which plots the mass (as a proportion of original mass) of an experimental sample against baking temperature (which can be thought of as a proxy for time), using both coal tar pitch and green binders. Although both types of pastes lose a similar amount of mass over the entire baking process, the loss with the green binder is faster than for the coal tar pitch binder. As this rate is highest around $100^{\circ} \mathrm{C}$, Elkem believes this is primarily due to the evaporation of water. They also believe that this evaporation is causing pressure to build up within the paste, leading to cracking. Experiments have confirmed that baking the new paste more slowly reduces the amount of cracking, consistent with this hypothesis.

In the experimental setup, a small cylindrical sample (diameter and height $5 \mathrm{~cm}$ ) is heated from all sides from room temperature to approximately $400^{\circ} \mathrm{C}$ over several hours. The external pressure is kept at atmospheric pressure, and the sample is free to expand or contract in all directions.

To build a mathematical model of ramming paste based on this experimental setup, we will treat the paste as a porous medium, where the voids are filled with water vapour (gas $g$ ). The solid skeleton will be a mixture of anthracite $(a)$ and binder (b). We will make the following assumptions. The binder is only comprised of free water (i.e. we will treat the organic compounds as part of the anthracite); the only reaction is the evaporation of water; the anthracite and binder move together; we are at local thermal equilibrium (i.e. no heat transfer between components in the same representative volume); the solid skeleton is isotropic; no solid deformations such as hardening or thermal expansion occur; the effects of mechanical energy and work are negligible compared to heat energy and latent heat of evaporation; and water vapour is an ideal gas.

There are several processes we wish to model, including heat transfer through the paste, evaporation of water, and flow of water vapour through the paste (a porous material).

\subsection{Conservation of Mass}

The model used here for flow and mechanical effects in a porous medium is based on the general framework from $[5,6$, 7,8 ], although we assume no solid deformations for sake of simplicity. In this medium, consider a representative volume $V$ (assumed to be significantly larger than the microstructure size). This volume is taken up by volumes of each constituent, $V=$ $V_{a}+V_{b}+V_{g}$, and has mass $m=m_{a}+m_{b}+m_{g}$. We normalise and consider the volume fractions of each constituent, $\phi_{k}:=V_{k} / V$ $(k \in\{a, b, g\})$, so $\phi_{a}+\phi_{b}+\phi_{g}=1$. These materials have an 
intrinsic density $\rho_{k}:=m_{k} / V_{k}(k \in\{a, b, g\})$ and, within the porous medium, an apparent density $m_{k} / V=\phi_{k} \rho_{k}$. If each material is moving with velocity $\mathbf{v}_{k}$, and is created at a rate $I_{k}$ per unit volume (of the whole medium), conservation of mass within the volume $V$ reads

$$
\frac{\partial m_{k}}{\partial t}+\nabla \cdot\left(m_{k} \mathbf{v}_{k}\right)=V I_{k}, \quad k \in\{a, b, g\} .
$$

Since the only reaction is the evaporation from the binder to water vapour (at an evaporation rate $I_{g}=-I_{b}$ ), we choose $I_{a}=$ 0 .

For the evaporation rate, we follow [9] and use an Arrheniustype law:

$$
I_{g}:=A_{1} M_{w} \exp \left(-\frac{A_{2}}{R T}\right) \phi_{b} \phi_{g},
$$

where $A_{1}$ is a reaction rate constant, $M_{w}$ is the molar mass of water, $A_{2}$ is the activation energy of the reaction, $R$ is the universal gas constant, and $T$ is the temperature. We take the activation energy to be the latent heat of evaporation, i.e. $A_{2}=L_{g} M_{w}$. The extra factors of $\phi_{b}$ and $\phi_{g}$ represent the fact that the evaporation rate depends on the size of the interface between liquid and gas; the particular form is taken from [10].

\subsection{Momentum Balance}

To conserve momentum, we consider the partial stresses from each component, body forces (gravity), and momentum transfer between the different components. For each phase, conservation of momentum is written as [7]

$$
\phi_{k} \rho_{k}\left(\frac{\partial \mathbf{v}_{k}}{\partial t}+\mathbf{v}_{k} \cdot \nabla \mathbf{v}_{k}\right)=\phi_{k} \rho_{k} \mathbf{g}+\tilde{\mathbf{p}}_{k}, \quad k \in\{a, b, g\}
$$

where $\tilde{\mathbf{p}}_{k}$ is the momentum transfer to $k$ from the other constituents.

Conservation of momentum in the system is then ensured by enforcing $\sum_{k} \tilde{\mathbf{p}}_{k}=\mathbf{0}$. We can simplify by summing (3) over all $k$,

$$
\sum_{k \in\{a, b, g\}} \phi_{k} \rho_{k}\left(\frac{\partial \mathbf{v}_{k}}{\partial t}+\mathbf{v}_{k} \cdot \nabla \mathbf{v}_{k}\right)=-\nabla p+\bar{\rho} \mathbf{g}
$$

where $\bar{\rho}=\sum_{k} \phi_{k} \rho_{k}$ is the medium average density.

There are two different sources of velocity in the paste - from deformation of the solid skeleton and from movement of gas. However, we neglect solid mechanics, hence deformation of the solid skeleton is assumed to be zero, and $\mathbf{v}_{a}=\mathbf{v}_{b}=\mathbf{0}$. The gas movement is assumed to be driven by Darcy flow through the (porous) solid skeleton, which in the absence of solid deformations is (e.g. [8])

$$
\mathbf{v}_{g}=-\frac{k}{\phi_{g} \mu}\left(\nabla p-\rho_{g} \mathbf{g}\right)
$$

where $k$ is the permeability of the solid skeleton, $\mu$ is the gas viscosity, and $\mathbf{g}$ is acceleration due to gravity. The form (5) assumes that the porous structure is isotropic - if not, we would have to replace the scalar $k$ by a permeability tensor.

\begin{tabular}{llr}
\hline Symbol & Description & Units \\
\hline$m_{k}$ & Mass of component & $\mathrm{kg}$ \\
$V_{k}$ & Representative volume of component & $\mathrm{m}^{3}$ \\
$V$ & Total representative volume & $\mathrm{m}^{3}$ \\
$\rho_{k}$ & Intrinsic density of component & $\mathrm{kg} / \mathrm{m}^{3}$ \\
$\phi_{k}$ & Volume fraction of component & 1 \\
$\mathbf{v}_{k}$ & Velocity of component & $\mathrm{m} / \mathrm{s}$ \\
$T$ & Temperature & $\mathrm{K}$ \\
$p$ & Gas pressure & $\mathrm{Pa}$ \\
\hline
\end{tabular}

Table 1: Variables in the baking model. Here $k \in\{a, b, g\}$.

We will assume that the water vapour is an ideal gas. That is, the relationship between gas pressure $p$, temperature and gas density is given by the ideal gas law

$$
p=\frac{\rho_{g} R T}{M_{w}} .
$$

Volume changes may also occur as the reaction progress changes the quantity of each component. Following [8],

$$
\frac{1}{V_{k}} \frac{\partial V_{k}}{\partial t}=\frac{1}{m_{k}} \frac{\partial m_{k}}{\partial t}, \quad k \in\{a, b\}
$$

For the gas component, we have

$$
\frac{1}{V_{g}} \frac{\partial V_{g}}{\partial t}=\frac{1}{p} \frac{\partial p}{\partial t}
$$

\subsection{Energy Balance}

As we are neglecting solid deformations, the relevant components of energy in the system are kinetic energy of all components, and thermal energy. The energy is transferred or dissipated via convection, conduction, latent heat of vaporization, and work from gravity. We also assume that all components are heated in parallel, so we are at local thermal equilibrium (that is, the temperature of the solids and gas is the same whenever they are in contact). This leaves us with the simplified conservation of energy equation

$$
\sum_{k \in\{a, b, g\}}\left(\phi_{k} \rho_{k} c_{p, k} \frac{\partial T}{\partial t}+\nabla \cdot\left(\phi_{k} \rho_{k} c_{p, k} T \mathbf{v}_{k}\right)\right)=\nabla \cdot(\bar{\lambda} \nabla T)-L_{g} I_{g},
$$

where $c_{p, k}$ is the specific heat of component $k, \bar{\lambda}$ is the effective thermal conductivity of the mixture, and $L_{g}$ is the latent heat of vaporisation for water. The effective thermal conductivity of the mixture is defined by

$$
\bar{\lambda}=\left(\sum_{k \in\{a, b, g\}} \frac{\phi_{k}}{\lambda_{k}}\right)^{-1},
$$

where $\lambda_{k}$ is the thermal conductivity of component $k$. The effective thermal conductivity relationship in (10) assumes that all constituents are heated in parallel, consistent with our assumption of local thermal equilibrium [11].

This completes the description of the model dynamics. A full list of variables is given in Table 1. The associated parameters are given in Table 2. Material parameters for graphite are 


\begin{tabular}{llrl}
\hline Symbol & Description & Typical Value(s) & Notes \\
\hline$M_{w}$ & Molecular weight of water/steam & $0.018 \mathrm{kgmol}^{-1}$ & - \\
$R$ & Universal gas constant & $8.314 \mathrm{JK}^{-1} \mathrm{~mol}^{-1}$ & - \\
$A_{1}$ & Arrhenius evaporation rate in (2) & $1.2 \times 10^{8} \mathrm{molm}^{-3} \mathrm{~s}^{-1}$ & Fit to data; see Section 3 \\
$A_{2}$ & Arrhenius activation energy in (2) & $4.07 \times 10^{4} \mathrm{Jmol}^{-1}$ & $L_{g} M_{w}$ \\
$c_{p, a}$ & Specific heat capacity of anthracite & $(0.72-1.27) \times 10^{3} \mathrm{JK}^{-1} \mathrm{~kg}^{-1}$ & {$[12$, Tab. 13-2] } \\
$c_{p, b}$ & Specific heat capacity of water & $(4.2-4.3) \times 10^{3} \mathrm{JK}^{-1} \mathrm{~kg}^{-1}$ & {$[13], 20-150^{\circ} \mathrm{C}$} \\
$c_{p, g}$ & Specific heat capacity of steam & $2 \times 10^{3} \mathrm{JK}^{-1} \mathrm{~kg}^{-1}$ & {$[13]$} \\
$\lambda_{a}$ & Thermal conductivity of anthracite & $50-120 \mathrm{Wm}^{-1} \times K^{-1}$ & {$[12$, Fig. 11-3] } \\
$\lambda_{b}$ & Thermal conductivity of water & $0.58 \mathrm{Wm}^{-1} \times K^{-1}$ & {$[14], 25^{\circ} \mathrm{C}$} \\
$\lambda_{g}$ & Thermal conductivity of steam & $(0.016-0.019) \mathrm{Wm}^{-1} \times K^{-1}$ & {$[14], 25-125^{\circ} \mathrm{C}$} \\
$L_{g}$ & Latent heat of water evaporation & $2.26 \times 10^{6} \mathrm{Jkg}^{-1}$ & {$[13]$} \\
$k$ & Permeability of solid skeleton & $1.0 \times 10^{-15} \mathrm{~m}^{2}$ & {$[9]$ with $\phi=0.05$} \\
$\mu$ & Viscosity of steam & $(1.2-2.4) \times 10^{-5} \mathrm{Pas}^{-2}$ & {$[15], 250-750^{\circ} \mathrm{F}$} \\
$g$ & Gravitational acceleration (magnitude) & $9.81 \mathrm{~ms}^{-2}$ & - \\
$\phi_{b, 0}$ & Initial binder volume fraction & - & See Table 3 \\
$\rho_{b, 0}$ & Initial binder density & - & See Table 3 \\
$V_{0}$ & Initial representative volume & - & See Table 3 \\
\hline
\end{tabular}

Table 2: Dimensional parameters in the baking model.

used as a proxy for anthracite. Binder values are for water, and the material parameters for the solid skeleton are estimated by using values for anthracite (graphite).

\subsection{Initial and Boundary Conditions}

In the experimental setup, a cylindrical paste sample is placed within a bed of granular coke (which allows heating to occur), where the external temperature and pressure are specified, and the paste is free to expand. All the boundary conditions therefore apply to the entire surface of the sample.

Firstly, the external pressure is held constant (at atmospheric pressure), and the associated boundary condition is

$$
p=p_{\text {ext }},
$$

everywhere on the boundary. Similarly, we will assume the temperature of the material is fixed everywhere on the boundary

$$
T=T_{\text {bake }}(t),
$$

where $T_{b a k e}$ is some known function. In the original experiments, the temperature was increased linearly from $T_{0}$ to $T_{f}$ over time $t_{f}$, so

$$
T_{\text {bake }}(t)=T_{0}+\left(T_{f}-T_{0}\right) \frac{t}{t_{f}} .
$$

We could alternatively say that heat flux on the boundary is proportional to the temperature jump between the paste and the air. However, this would require using a parameter we do not know, so we refrain and use (12).

For initial conditions, we have

$$
T=T_{0}, \quad V=V_{0}, \quad \rho_{k}=\rho_{k, 0}, \quad \phi_{k}=\phi_{k, 0} \text { and } p=p_{\text {ext }} .
$$

The extra parameter values needed for initial and boundary conditions are given in Table 3. To calculate the initial volume fractions, we use the initial mass fractions, as calculated by Elkem Carbon. In general, the paste is approximately $85 \%$ anthracite, $5 \%$ water and $10 \%$ organic compounds (by mass). However, the experimental data provided by Elkem showed an estimated mass loss from water evaporation of $6.33 \%$. Therefore we will consider the paste to have mass fractions $S_{a, m}=$ 0.9367 and $S_{b, m}=0.0633$, where we have counted the mass of organic compounds as anthracite, since these compounds are unreactive in our model. Using the densities in Table 3, this is equivalent to the volume fractions

$$
S_{a, v}=\frac{S_{a, m} / \rho_{a, 0}}{S_{a, m} / \rho_{a, 0}+S_{b, m} / \rho_{b, 0}}, \quad S_{b, v}=\frac{S_{b, m} / \rho_{b, 0}}{S_{a, m} / \rho_{a, 0}+S_{b, m} / \rho_{b, 0}} .
$$

That is, we estimate the paste to be $86.7 \%$ anthracite and $13.3 \%$ water (by volume). To initiate the evaporation, we start with nonzero porosity, hence we will assume a volume fraction of $1 \%$ steam in the sample. We adjust the volume fractions of the anthracite and water appropriately, and get $\phi_{a, 0}=S_{a, v}\left(1-\phi_{g, 0}\right)$ and $\phi_{b, 0}=S_{b, v}\left(1-\phi_{g, 0}\right)$.

\subsection{Non-Dimensional Model}

To better understand the model, we now non-dimensionalise and simplify the model. We scale our variables as $\mathbf{x}=L \tilde{\mathbf{x}}$, $t=\tau \tilde{t}, V_{(k)}=V_{0} \tilde{V}_{(k)}, \rho_{k}=\rho_{k, 0} \tilde{\rho}_{k}, m_{k}=\left(\rho_{k, 0} V_{0}\right) \tilde{m}_{k}, \mathbf{v}_{k}=$ $\nu \tilde{\mathbf{v}}_{k}, p=\Pi \tilde{p}, T=T_{0}+\left(T_{f}-T_{0}\right) \tilde{T}, \bar{\lambda}=\lambda_{0} \tilde{\lambda}$. Similarly, we write gravitational acceleration as $\mathbf{g}=g \mathbf{e}_{g}$ for a unit vector $\mathbf{e}_{g}$. Scaling values not previously defined in Tables 2 or 3 are given in Table 4. Of particular importance in the scalings is that we choose the velocity scaling $v:=\Pi k /(\mu L)$, where $\Pi:=p_{\text {ext }}$ is the pressure scaling. We then choose our time scale $\tau:=L / v=$ $\mu L^{2} /(\Pi k)=123.4 \mathrm{~s}$ to be the time scale given by the movement of gas.

The non-dimensional parameter groups which arise are listed in Table 5. We are able to make the following simplifications: (i) ignore the contribution of gravity in (5), as $\mathcal{G}_{g}$ is small; (ii) 


\begin{tabular}{llrl}
\hline Symbol & Description & Typical Value(s) & Notes \\
\hline$L$ & Radius of sample & $0.025 \mathrm{~m}$ & Experiment \\
$h$ & Height of sample & $0.05 \mathrm{~m}$ & Experiment \\
$V_{0}$ & Initial representative volume size & - & Not used \\
$T_{0}$ & Initial temperature & $293.15 \mathrm{~K}$ & Experiment \\
$T_{f}$ & Final temperature & $673.15 \mathrm{~K}$ & Experiment \\
$t_{f}$ & Baking time & $2.88 \times 10^{4} \mathrm{~s}$ & Experiment \\
$\rho_{a, 0}$ & Initial density of anthracite & $2.26 \times 10^{3} \mathrm{kgm}^{-3}$ & {$[12$, Table 1-1] } \\
$\rho_{b, 0}$ & Initial density of water & $0.998 \times 10^{3} \mathrm{kgm}^{-3}$ & {$[13]$} \\
$\rho_{g, 0}$ & Initial density of steam & $0.749 \mathrm{kgm}^{-3}$ & $(6)$ at $T_{0}, p_{e x t}$ \\
$p_{e x t}$ & External gas pressure & $1.013 \times 10^{5} \mathrm{~Pa}$ & - \\
$S_{a, m}$ & Mass fraction of anthracite in paste & 0.9367 & Experiment \\
$S_{b, m}$ & Mass fraction of binder in paste & 0.0633 & Experiment \\
$\phi_{a, 0}$ & Initial volume fraction (anthracite) & 0.858 & $(15)$ \\
$\phi_{b, 0}$ & Initial volume fraction (water) & 0.132 & $(15)$ \\
$\phi_{g, 0}$ & Initial volume fraction (gas) & 0.01 & See text \\
\hline
\end{tabular}

Table 3: Dimensional and non-dimensional parameters in the initial and boundary conditions of the baking model.

\begin{tabular}{llrl}
\hline Symbol & Description & Typical Value(s) & Notes \\
\hline$\Pi$ & Pressure scale & $1.013 \times 10^{5} \mathrm{~Pa}$ & $\Pi:=p_{\text {ext }}$ \\
$v$ & Velocity scaling & $2.010^{-4} \mathrm{~ms}^{-1}$ & $v:=\Pi k /(\mu L)$ \\
$\tau$ & Time scale & $123.4 \mathrm{~s}$ & $\tau:=L / v$ \\
$\lambda_{0}$ & Thermal conductivity scaling & $1.16 \mathrm{Wm}^{-1} \mathrm{~K}^{-1}$ & $(10)$ evaluated at $t=0$ \\
\hline
\end{tabular}

Table 4: Scaling variables which have not been previously defined in Tables 2 or 3.

\begin{tabular}{llr}
\hline Symbol & Relationship to parameters & Typical Value(s) \\
\hline$\tilde{A}_{2}$ & $A_{2} /\left(R T_{0}\right)$ & 16.7 \\
$C_{b}$ & $\rho_{b, 0} c_{p, b} /\left(\rho_{a, 0} c_{p, a}\right)$ & 1.9 \\
$C_{g}$ & $\rho_{g, 0} c_{p, g} /\left(\rho_{a, 0} c_{p, a}\right)$ & $6.6 \times 10^{-4}$ \\
$\mathrm{Fo}_{a}$ & $\lambda_{0} \tau /\left(\rho_{a, 0} c_{p, a} L^{2}\right)$ & 0.10 \\
$\mathcal{G}_{g}$ & $\rho_{g, 0} g L / \Pi$ & $1.8 \times 10^{-6}$ \\
$\mathcal{I}$ & $\rho_{g, 0} R T_{0} /\left(\Pi M_{w}\right)$ & 1.0 \\
$\mathcal{J}_{b}$ & $A_{1} M_{w} \tau / \rho_{b, 0}$ & $2.7 \times 10^{5}$ \\
$\mathcal{J}_{g}$ & $A_{1} M_{w} \tau / \rho_{g, 0}$ & $3.6 \times 10^{8}$ \\
$\tilde{P}_{b}$ & $\rho_{b, 0} / \rho_{a, 0}$ & 0.44 \\
$\tilde{P}_{g}$ & $\rho_{g, 0} / \rho_{a, 0}$ & $3.3 \times 10^{-4}$ \\
$\mathrm{St}$ & $c_{p, b}\left(T_{f}-T_{0}\right) / L_{g}$ & 0.7 \\
$\Delta_{T}$ & $\left(T_{f}-T_{0}\right) / T_{0}$ & 1.3 \\
$\tau_{f}$ & $\tau / t_{f}$ & $4.3 \times 10^{-3}$ \\
\hline
\end{tabular}

Table 5: Non-dimensional parameter groups.

ignore terms from inertia, gas pressure, and gravity in (4), as these are negligibly small; (iii) ignore the heat energy stored in the gas and the advection of heat by gas flow in (9), as both $C_{g}$ and $C_{g} / \Delta_{T}$ are small. The non-dimensional model is then given by:

$$
\begin{gathered}
\tilde{V}=\tilde{V}_{a}+\tilde{V}_{b}+\tilde{V}_{g}, \phi_{k}=\frac{\tilde{V}_{k}}{\tilde{V}}, \tilde{\rho}_{k}=\frac{\tilde{m}_{k}}{\tilde{V}_{k}}, k \in\{a, b, g\} \\
\frac{\partial \tilde{m}_{a}}{\partial \tilde{t}}=0, \frac{\partial \tilde{m}_{b}}{\partial \tilde{t}}=-\mathcal{J}_{b} \tilde{V}_{v} \tilde{I}_{v}, \frac{\partial \tilde{m}_{g}}{\partial \tilde{t}}+\nabla \cdot\left(\tilde{m}_{g} \tilde{\mathbf{v}}_{g}\right)=\mathcal{J}_{g} \tilde{V} \tilde{I}_{v} \\
\tilde{I}_{v}:=\exp \left(-\frac{\tilde{A}_{2}}{1+\Delta_{T} \tilde{T}}\right) \phi_{b} \phi_{g}
\end{gathered}
$$

$$
\begin{gathered}
\tilde{\mathbf{v}}_{g}=-\frac{1}{\phi_{g}} \nabla \tilde{p}, \\
\tilde{p}=I_{\tilde{\rho}_{g}}\left(1+\Delta_{T} \tilde{T}\right), \\
\frac{1}{\tilde{V}_{k}} \frac{\partial \tilde{V}_{k}}{\partial \tilde{t}}=\frac{1}{\tilde{m}_{k}} \frac{\partial \tilde{m}_{k}}{\partial \tilde{t}}, k \in\{a, b\}, \\
\frac{1}{\tilde{V}_{g}} \frac{\partial \tilde{V}_{g}}{\partial t}=\frac{1}{\tilde{p}} \frac{\partial \tilde{p}}{\partial \tilde{t}}, \\
\left(\phi_{a} \tilde{\rho}_{a}+C_{b} \phi_{b} \tilde{\rho}_{b}\right) \frac{\partial \tilde{T}}{\partial \tilde{t}}=\mathrm{Fo}_{a} \nabla \cdot(\tilde{\lambda} \nabla \tilde{T})-\frac{C_{b} \mathcal{J}_{b} \tilde{I}_{v}}{\mathrm{St}} .
\end{gathered}
$$

The boundary conditions, which apply to the entire surface of the sample, are

$$
\tilde{p}=1 \quad \text { and } \quad \tilde{T}=\tilde{T}_{b a k e}(\tilde{t}):=\tau_{f} \tilde{t},
$$

since $\tilde{\Pi}$ and $\mathcal{G}_{0}$ are small. Lastly, the initial conditions are:

$$
\tilde{T}=0, \quad \tilde{V}=1, \quad \tilde{\rho}_{k}=1, \quad \phi_{k}=\phi_{k, 0} \quad \text { and } \quad \tilde{p}=1
$$

\section{Numerical Results and Discussion}

In this section we will discuss the results of numerical simulations of the model outlined in Section 2, implemented using COMSOL. To give better understanding of the relevant physics, and allow for greater validation, a simplified version of the model was implemented. Although this 'simplified model' is substantially reduced, it keeps enough of the key processes to produce useful results and analysis for understanding the baking process. We begin by outlining this simpler model. 

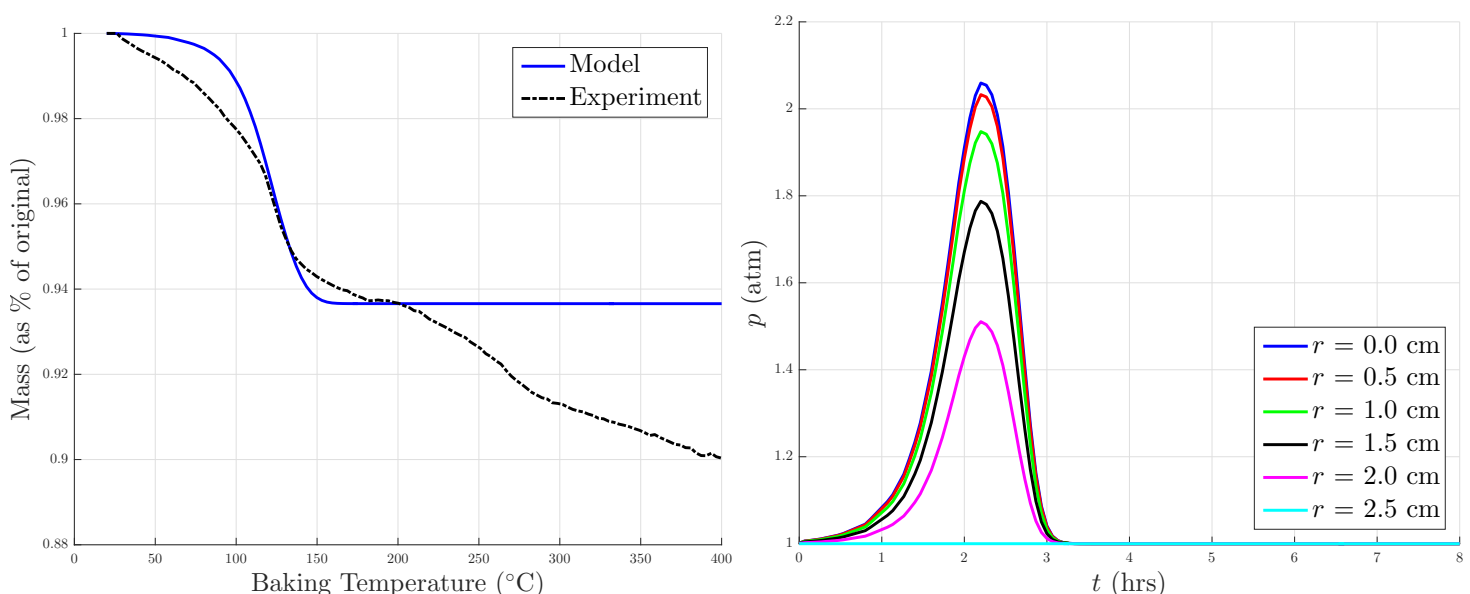

Figure 3: Numerical simulations for the model presented in Section 3.1 using all default parameter values. The sample mass is compared to experimental data (source: Elkem Carbon).

\subsection{Simplified Heat and Mass Transfer Model}

To simplify the model outlined in Section 2, we assume the solid volume $V$ remains constant, and use this condition instead of (8). Therefore, conservation of mass can be simplified to (in dimensional form)

$$
\frac{\partial\left(\phi_{a} \rho_{a}\right)}{\partial t}=0, \frac{\partial\left(\phi_{b} \rho_{b}\right)}{\partial t}=-I_{g}, \frac{\partial\left(\phi_{g} \rho_{g}\right)}{\partial t}+\nabla \cdot\left(\phi_{g} \rho_{g} \mathbf{v}_{g}\right)=I_{g}
$$

However, from (7), we may conclude that both $\phi_{a}$ and $\rho_{b}$ are constant (in time). To better align with COMSOL's in-built physics engines, we introduce a new variable

$$
c_{b}:=\frac{\phi_{b} \rho_{b}}{M_{w}},
$$

which is the concentration of binder per representative volume of the whole porous medium (with units $\mathrm{mol} \mathrm{m}^{-3}$ ).

Introducing the evaporation rate written in terms of concentration

$$
I_{v}:=\frac{I_{g}}{M_{w}}=A_{1} \exp \left(-\frac{A_{2}}{R T}\right) \phi_{b} \phi_{g}
$$

our mass conservation relations are reduced to

$$
\frac{\partial c_{b}}{\partial t}=-I_{v} \quad \text { and } \quad \frac{\partial\left(\phi_{g} \rho_{g}\right)}{\partial t}+\nabla \cdot\left(\phi_{g} \rho_{g} \mathbf{v}_{g}\right)=M_{w} I_{v}
$$

together with constant $\phi_{a}, \rho_{a}$ and $\rho_{b}$. The volume fraction $\phi_{b}$ can then be calculated from (27), and we also know $\phi_{g}=$ $1-\phi_{a}-\phi_{b}$. The model is completed with Darcy's law (5) (but neglecting gravity as per (19)), the ideal gas law (6) and conservation of energy (9) (neglecting convection as per (23))

$$
\mathbf{v}_{g}=-\frac{1}{\phi_{g}} \frac{k}{\mu} \nabla p,\left(\sum_{k \in\{a, b, g\}} \rho_{k} c_{p, k}\right) \frac{\partial T}{\partial t}=\nabla \cdot(\bar{\lambda} \nabla T)-L_{g} M_{w} I_{v},
$$

with $p=\frac{\rho_{g} R T}{M_{w}}$. There are no changes to the boundary or initial conditions.

\subsection{Numerical Results for the Simplified Heat and Mass Trans- fer Model}

The results from the 'simplified model' presented in Section 3.1 were calculated in COMSOL. In Figure 3, the value of different variables is sampled at several points along a radial line halfway up the cylinder (so $r=0$ is the very centre of the sample, and $r=2.5 \mathrm{~cm}$ is the boundary of the sample).

First we consider the total mass loss of the sample, which is due to the outward flux of gas, shown in Figure 3. In keeping with Figure 1, it is plotted against baking temperature $T_{\text {bake }}$ rather than time. The model predicts mass loss which closely aligns with the experimental data (which has been normalised so that the first observation after baking commences corresponds to no mass loss). The model predicts that the majority of the mass loss occurs in a small window, after which all the water has evaporated, so no further loss occurs. This matches the experimental data until the baking temperature reaches $200 \mathrm{C}$. After this point, the mass loss is driven by other volatile chemicals not included in this model, so the divergence of model and experiment from here is expected. It is of particular note that the timing and rate of peak mass loss, at around $120 \mathrm{C}$ is captured very well. This is the period of most concern, since it corresponds to maximum pressure, and therefore highest likelihood of cracking. Note that the reaction rate parameter $A_{1}$ has been approximately calibrated to the experimental data in Figure 3.

Figure 3 also shows the variation in gas pressure $p$. Initially the pressure remains close to its initial level, but increases as the baking temperature increases and the evaporation reaction proceeds. It reaches a maximum value after slightly more than 2 hours, before rapidly dropping to external (atmospheric) pressure, as dictated by the boundary conditions. The pressure is highest in the centre of the sample, but the pressure gradient is higher nearer the boundary.

The temperature profile within the sample is close to constant (in space), as conduction occurs faster than the increase in baking temperature. The centre of the sample is cooler when the evaporation is happening fastest - due to the latent heat of vaporisation - but this different is small compared to the overall temperature increase. 

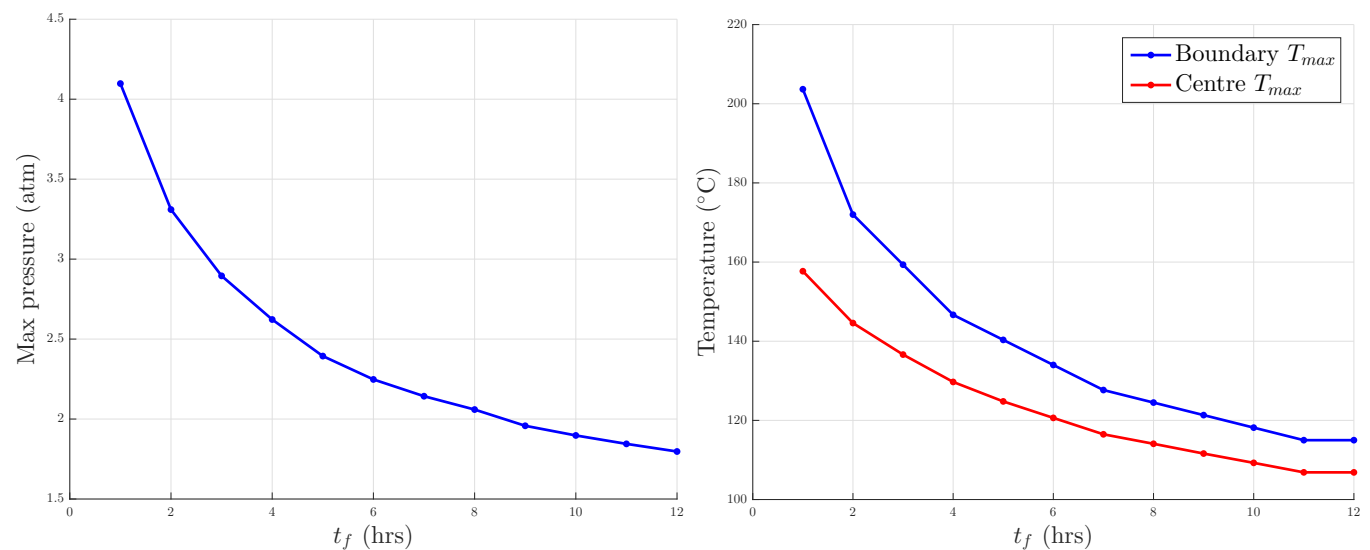

Figure 4: Dependency of maximum pressure and $T_{\max }$ on total baking time $t_{f}$.
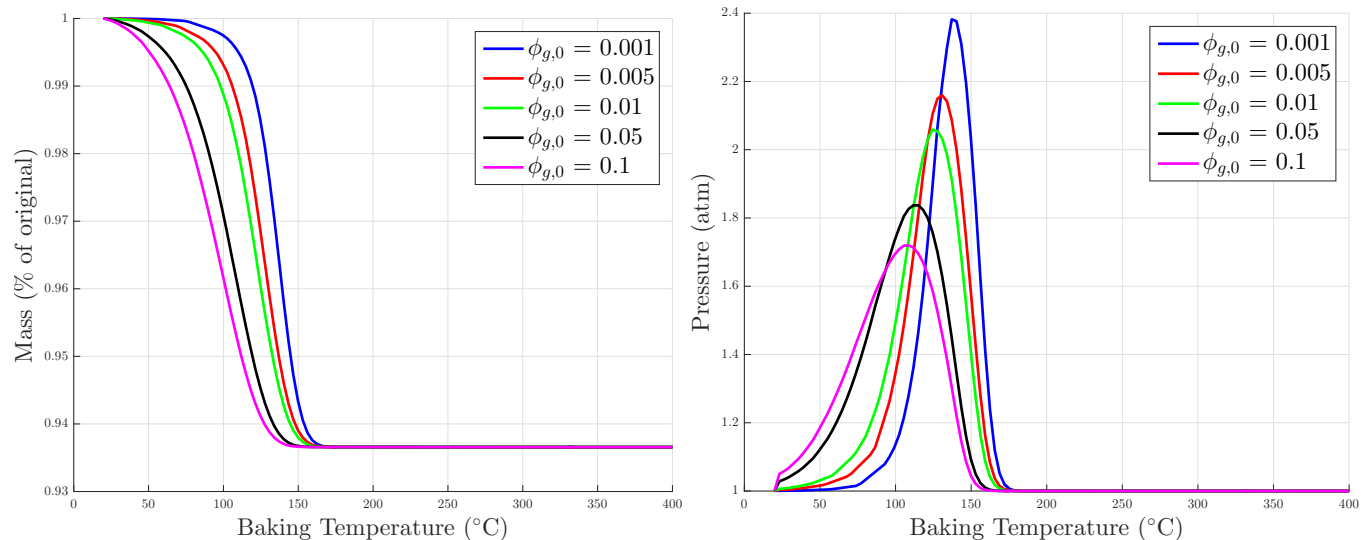

Figure 5: Impact of varying $\phi_{g, 0}$ on sample mass and maximum pressure.

We will now consider how the model behaves as certain parameters are changed. Since we are most concerned about the appearance of cracking, the main quantity of interest will be the maximum pressure attained anywhere in the sample, at any point in time. From Figure 3, this maximum will likely be attained in the centre of the sample. However, as the paste hardens as the baking progresses, we will also consider the temperature at the time when the maximum pressure is attained, $T_{\max }$. We expect that the larger $T_{\max }$, the further the baking has progresses when the maximum pressure is attained, so the paste can withstand a larger pressure before cracking.

\subsubsection{Sensitivity to Baking Time}

The results of varying total baking time $t_{f}$ from 1 to 12 hours are shown in Figure 4. The temperature range for baking is always the same, so this corresponds with slowing the rate at which the external temperature is increased from $T_{0}$ to $T_{f}$. The left plot in Figure 4 shows the maximum pressure attained anywhere in the sample (at any point in the baking process). The right plot in Figure 4 shows both the external baking temperature and temperature in the centre of the sample when this maximum pressure was attained, $T_{\max }$.

With longer baking time, the temperature throughout the sample, and hence the evaporation rate, increases more slowly. Therefore it is not surprising that in Figure 4, we see that a longer baking time is associated with lower pressures within the sample. We also see that the maximum pressure is attained at lower baking temperatures. This is because once the temperature increases sufficiently to start the evaporation occurring quickly, it remains at that (moderate) temperature for longer, so the reaction proceeds at that fast rate for more time.

Separately, we can also see that the core and boundary temperatures are closer when the total baking time is slower. This is to be expected, as when the baking time is longer, the time scale for conduction is faster (relative to the time scale of external temperature changes).

\subsubsection{Sensitivity to Initial Porosity}

The value $\phi_{g, 0}=0.01$ used in the above results was chosen as a sensible small value, but not calibrated to experimental data. To examine the impact of this assumption, Figure 5 shows mass loss and maximum pressure (within the sample) as a function of baking temperature. The other volume fractions were initialised as per (15), with $S_{a, m}=0.9367$ and $S_{b, m}=0.0633$ as before. As shown in Figure 5, increasing the initial porosity causes mass loss in the sample to begin sooner, and take longer to complete. We also find that higher initial porosity means the maximum pressure occurs earlier, but at a much lower level. The variations in the pressure profile are large, so it is therefore important to accurately estimate $\phi_{g, 0}$. 

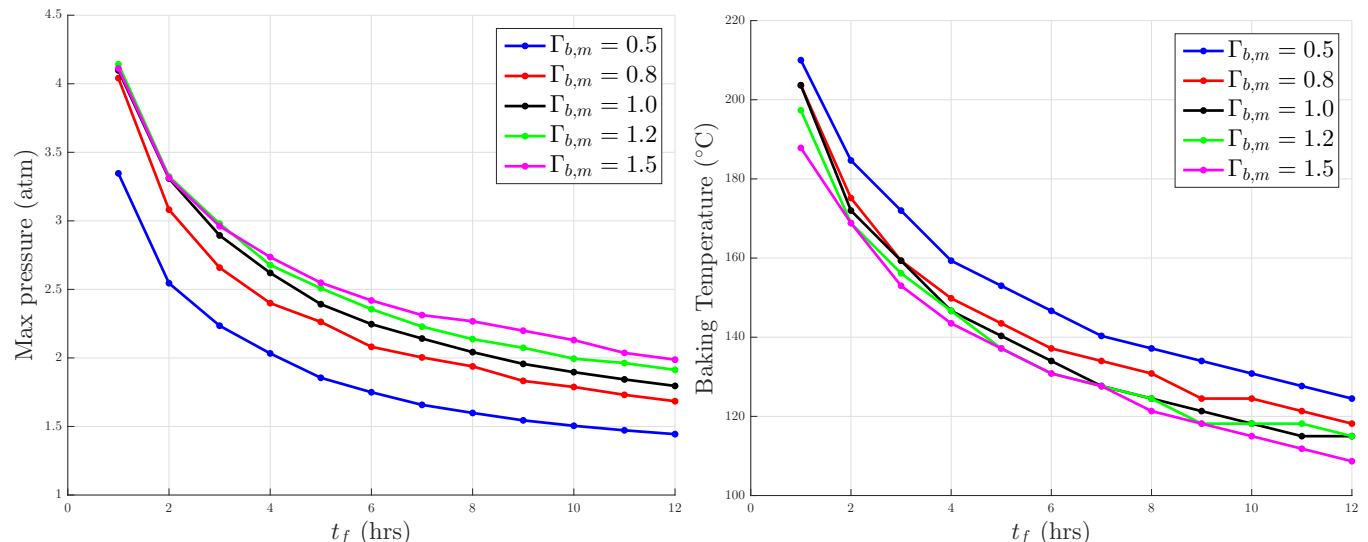

Figure 6: Impact of varying the anthracite to binder ratio on maximum pressure and boundary $T_{\max }$.
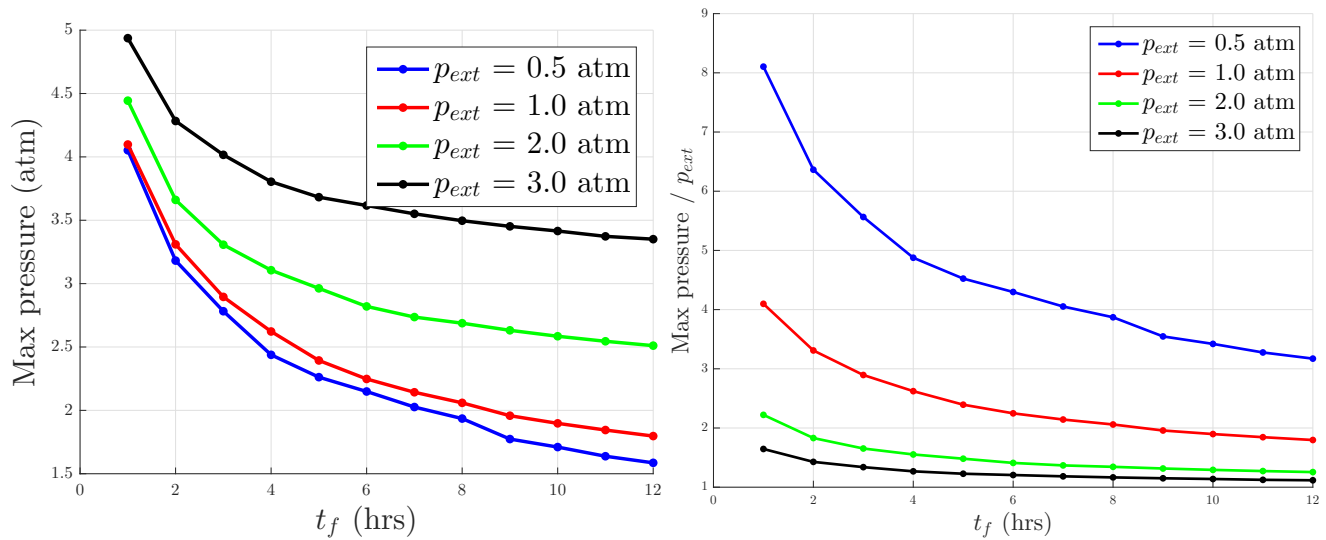

Figure 7: Impact of varying the external pressure $p_{\text {ext }}$ on the maximum pressure in the sample.

\subsubsection{Sensitivity to Anthracite to Binder Ratio}

One value that Elkem is potentially able to modify in testing is the relative fraction of water to other substances in the paste. To test the sensitivity to this, we scaled the mass fraction of water as $S_{b, m}=0.0633 \Gamma_{b, m}$ for different values of $\Gamma_{b, m}$, and recalculated $\phi_{a, 0}$ and $\phi_{b, 0}$ using (15). Throughout we continued to use the original value $\phi_{g, 0}=0.01$. The sensitivity of maximum pressure and boundary $T_{\max }$ to moves in $t_{f}$ and $\Gamma_{b, m}$ are shown in Figure 6.

Firstly, we notice that decreasing the proportion of water (or increasing the amount of anthracite to organic compounds; decreasing $\Gamma_{b, m}$ ) reduces the pressure in the sample. For instance, if we halved the mass fraction of water in the paste, a 4 hour bake would achieve the same pressure as an 8 hour bake does currently. Interestingly, reducing the amount of water in the paste would simultaneously give a higher $T_{\text {max }}$; that is, the pressure is highest further in the baking process, when the paste has hardened more. Therefore we could expect that modifying the amount of water in the paste could substantially change the amount (or likelihood) of cracking in the sample.

\subsubsection{Sensitivity to External Pressure}

Another parameter of interest to Elkem is the external pressure $p_{\text {ext }}$, which they are able to control experimentally. Currently set to be atmospheric pressure, it appears in both the initial (14) and boundary conditions (11) of the model. The results of varying $p_{\text {ext }}$ are shown in Figure 7.

Firstly, the model never predicts pressures lower than $p_{\text {ext }}$. Unsurprisingly therefore, the maximum pressure attained is higher when $p_{\text {ext }}$ is higher too (as in Figure 7). However, if we scale the maximum pressure by $p_{\text {ext }}$, we see in Figure 7 that the maximum pressure increases more slowly than $p_{\text {ext }}$.

The boundary $T_{\max }$ is not sensitive to variations in $p_{\text {ext }}$; i.e. the maximum pressure occurs at similar times in the baking process, regardless of $p_{\text {ext }}$. This is likely because our evaporation model does not include any pressure dependency.

It is unclear from this information whether a higher or lower value of $p_{\text {ext }}$ is better for reducing cracking. This will depend on whether the stresses which cause cracking are based on the absolute level of the internal pressure, or the relative increase in internal pressure versus external pressure.

\subsection{Nonlinear Baking Profile}

A key feature of the experimental and numerical results presented in Section 3.2 is that most of the changes occur over a relatively short time. We also have observed from Section 3.2.1 that a longer baking time is associated with lower pressure within the sample. Therefore, perhaps if we slow the baking only in the temperature range where most of the changes occur, we may achieve lower pressure while still maintaining a short total baking time. 

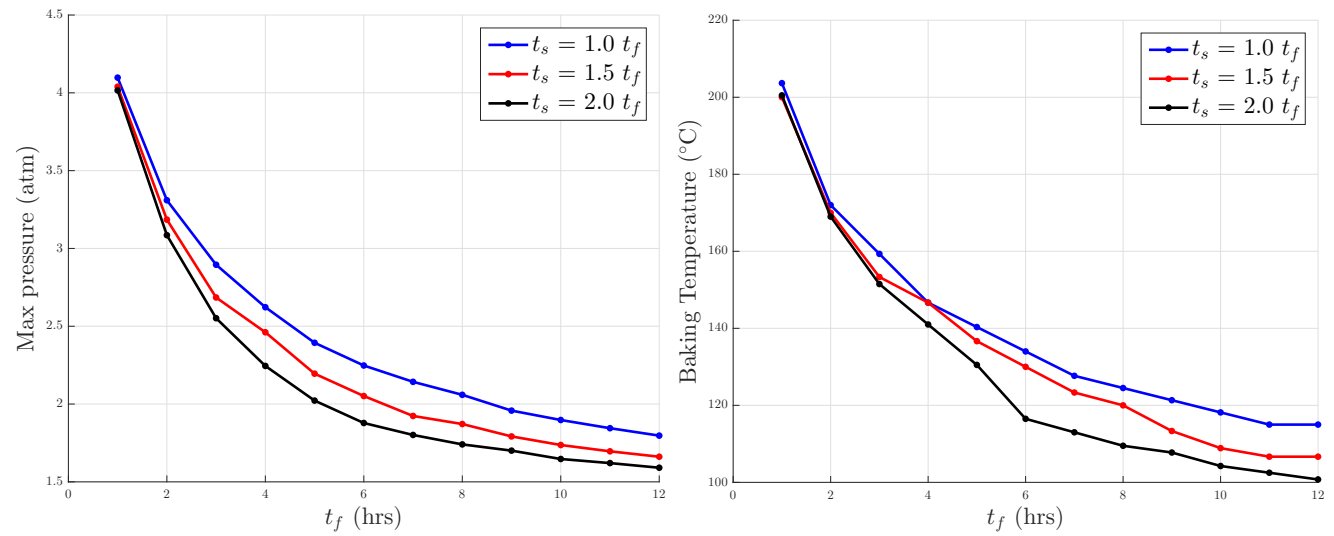

Figure 8: Dependency of maximum pressure and boundary $T_{\max }$ on times $t_{f}$ and $t_{s}$, when using the piecewise linear baking profile (31) with $d T=20 \mathrm{C}$.
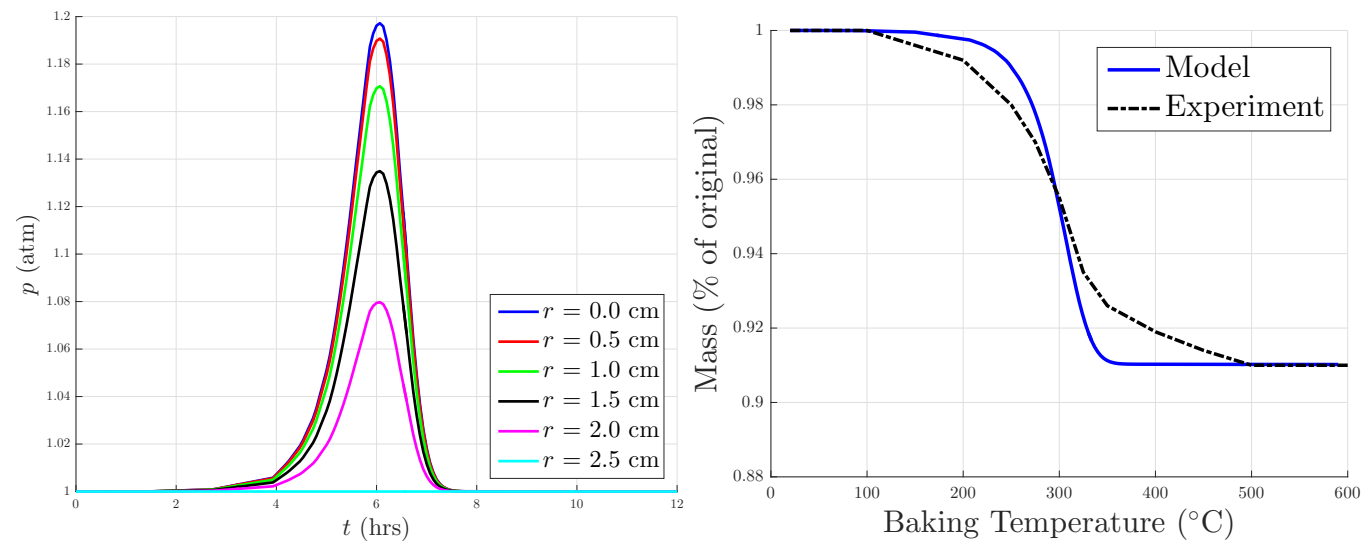

Figure 9: Results of the numerical model presented in Section 3.1 adapted for coal tar pitch binders, using parameter values from Table 6.

To test this, we changed the external baking temperature $T_{\text {bake }}$ in (12) from a linear profile (13) to a piecewise linear profile. The sample is heated from $T_{0}$ to $100 \mathrm{C}-d T$ and from $100 \mathrm{C}+d T$ to $T_{f}$ at a rate given by total baking time $t_{f}$. In between, the sample is heated from $100 \mathrm{C}-d T$ to $100 \mathrm{C}+d T$ at a rate given by total baking time $t_{s} \geq t_{f}$. That is, we have

$$
T_{\text {bake }}(t)= \begin{cases}T_{0}+\left(\frac{T_{f}-T_{0}}{t_{f}}\right) t, & 0 \leq t \leq t_{1}, \\ (100-d T)+\left(\frac{T_{f}-T_{0}}{t_{s}}\right)\left(t-t_{1}\right), & t_{1} \leq t \leq t_{2}, \\ (100+d T)+\left(\frac{T_{f}-T_{0}}{t_{f}}\right)\left(t-t_{2}\right), & t_{2} \leq t \leq t_{3},\end{cases}
$$

where

$$
\begin{gathered}
t_{1}:=\frac{100 \mathrm{C}-d T-T_{0}}{T_{f}-T_{0}} t_{f}, \quad t_{2}:=t_{1}+\frac{2 d T}{T_{f}-T_{0}} t_{s}, \\
t_{3}:=t_{2}+\frac{T_{f}-100 \mathrm{C}-d T}{T_{f}-T_{0}} t_{f} .
\end{gathered}
$$

The total baking time is $t_{3} \geq t_{f}$. The results of varying $t_{f}$ and $t_{s}$ are shown in Figure 8. A value of $d T=20 \mathrm{C}$ was used throughout.

Considering Figure 8, we see that reducing the baking rate just over the $80-120 \mathrm{C}$ range is sufficient to lead to a substantial reduction in pressure. Using this approach for baking could therefore mean either a lower risk of cracking for the same total baking time, or the same risk of cracking in a shorter total baking time. For example, by heating half as quickly in this critical range $\left(t_{s}=2 t_{f}\right)$ gives a similar maximum pressure to the original 8 hour experiment with a 'fast' baking time $t_{f}$ of only 5 hours (or a total baking time of 5.5 hours). However, there is a trade-off shown in Figure 8 - using a slower baking speed in the critical region, the maximum pressure is attained at lower temperatures than it would otherwise.

\subsection{Coal Tar Pitch}

The above work has focused on modelling Elkem's ramming paste using green binders. However, we also have mass loss data for paste using industry-standard coal tar pitch binder. As shown in Figure 1, paste with a coal tar pitch binder loses its mass more slowly, and at higher temperatures, than with organic binders. Here we show how the simplified model from Section 3.1 performs when applied to coal tar pitch binders. The changes to parameters required are listed in Table 6. Based on the model in [9], we assume that the binder is solely comprised on chrysene $\left(\mathrm{C}_{18} \mathrm{H}_{12}\right)$. For the thermal properties of the gas component, we use the relevant values for air.

In Figure 9, we see a similar profile of internal pressures over time, with a similar radial variation, to the organic binder case (see Figure 3). However, the maximum pressure reached is much lower. This suggests that the coal tar pitch binders are less prone to cracking over a similar baking time, which agrees with Elkem's observations. The mass loss predicted by the model is compared to experimental data in Figure 9. The fit is not as close as for organic binders (see Figure 3), which is 


\begin{tabular}{llrl}
\hline Symbol & Description & Typical Value(s) & Notes \\
\hline$M_{w}$ & Molecular weight of gas (\& binder) & $0.228 \mathrm{~kg} \mathrm{~mol}^{-1}$ & {$[9]$, chrysene } \\
$A_{1}$ & Arrhenius evaporation rate in (2) & $1.2 \times 10^{6} \mathrm{~mol} \mathrm{~m}^{-3} \mathrm{~s}^{-1}$ & Fit to data \\
$A_{2}$ & Arrhenius activation energy in (2) & $5.11 \times 10^{4} \mathrm{~J} \mathrm{~mol}^{-1}$ & {$[9]$, chrysene } \\
$c_{p, b}$ & Specific heat capacity of binder & $1.47 \times 10^{3} \mathrm{~J} \mathrm{~K}^{-1} \mathrm{~kg}^{-1}$ & {$[16]$, tar } \\
$c_{p, g}$ & Specific heat capacity of gas & $1.01 \times 10^{3} \mathrm{~J} \mathrm{~K}^{-1} \mathrm{~kg}^{-1}$ & {$[17]$, air } \\
$\lambda_{b}$ & Thermal conductivity of binder & $1.4 \mathrm{~W} \mathrm{~m}^{-1} \mathrm{~K}^{-1}$ & Estimated from Elkem data \\
$\lambda_{g}$ & Thermal conductivity of gas & $3 \times 10^{-2} \mathrm{~W} \mathrm{~m}^{-1} \mathrm{~K}^{-1}$ & {$[17]$, air } \\
$L_{g}$ & Latent heat of vaporisation & $1.18 \times 10^{6} \mathrm{~J} \mathrm{~kg}^{-1}$ & {$[9]$, tar } \\
$\mu$ & Viscosity of gas & $2.3 \times 10^{-5} \mathrm{~Pa} \mathrm{~s}^{-1}$ & {$[18]$, air } \\
$\rho_{b, 0}$ & Initial density of binder & $1.274 \times 10^{3} \mathrm{~kg} \mathrm{~m}^{-3}$ & {$[18$, p. 3-128], chrysene } \\
$\rho_{g, 0}$ & Initial density of gas & $1.2 \mathrm{~kg} \mathrm{~m}^{-3}$ & {$[17]$, air } \\
$S_{b, m}$ & Initial mass fraction (binder in paste) & 0.09 & Experiment \\
$\phi_{a, 0}$ & Initial volume fraction (anthracite) & 0.842 & $(15)$ \\
$\phi_{b, 0}$ & Initial volume fraction (binder) & 0.148 & $(15)$ \\
\hline & & &
\end{tabular}

Table 6: Model parameters used for coal tar pitch binder simulations.

unsurprising - there are many more reactions and species that are important in the baking of coal tar pitch. Compared to the organic binder case, the simplification to a single reaction is less appropriate.

Note that to provide the best comparison to experimental data, we have used the same $T_{f}$ and $t_{f}$ parameters as before, and hence the same function $T_{\text {bake }}(13)$, but run the simulation for times beyond $t_{f}=8 \mathrm{hrs}$. This means we can reach baking temperatures beyond $T_{f}=400 \mathrm{C}$, where a reasonable part of the mass loss occurs.

To gain a greater understanding of the model's application to coal tar pitch binders, Figure 10 shows the results of varying the baking speed (via varying $t_{f}$ in (13)). As before, a slower baking speed results in lower pressures within the sample, and these pressures are all significantly lower than the corresponding values for organic binders (see Figure 4). Again, these maximum pressures are reached at lower temperatures with slower baking speeds.

A noticeable difference here compared to the green binders is that the gap between baking temperature and central $(r=0)$ temperature is much smaller than for green binders (see Figure 4). This is due to the higher thermal conductivity and lower specific heat of the coal tar pitch compared to water. With the values from Table 6 , the new non-dimensional parameters, relevant to this phenomenon, are $C_{b}=0.57$ and $\mathrm{Fo}_{a}=0.20$. Considering (23) and comparing to the values for green binders in Table 5, this means that heat conduction is more important for the coal tar pitch binder, so we would expect the internal temperature variation to be smaller.

\subsection{Simplified Model Incorporating Thermal Expansion}

It would be natural at this stage to begin extending the model to incorporate the mechanical aspects of the full model from Section 2. As a first step, in this section we present an extension of the simplified model from Section 3.1 to include thermal expansion. To this end, instead of assuming no displacement or volume changes, we instead replace (7)-(8) with

$$
\begin{aligned}
& \frac{1}{V_{k}} \frac{\partial V_{k}}{\partial t}=\alpha_{k} \frac{\partial T}{\partial t}+\frac{1}{m_{k}} \frac{\partial m_{k}}{\partial t}, \quad k \in\{a, b\}, \\
& \frac{1}{V_{g}} \frac{\partial V_{g}}{\partial t}=\frac{1}{p} \frac{\partial p}{\partial t}-\frac{1}{\phi_{g}}\left(\phi_{a} \alpha_{a}+\phi_{b} \alpha_{b}\right) \frac{\partial T}{\partial t} .
\end{aligned}
$$

Here $\alpha_{k}$ is the relevant coefficient of thermal expansion (with typical values $\alpha_{a}=7.5 \times 10^{-6} \mathrm{~K}^{-1}$ [12, Fig. 10-1] and $\alpha_{b}=$ $\left.(2.1-9.8) \times 10^{-4} \mathrm{~K}^{-1}[13]\right)$. We make needed simplifications to get

$$
\begin{aligned}
\rho_{k} & =\rho_{k, 0} e^{-\alpha_{k}\left(T-T_{0}\right)}, \quad k \in\{a, b\}, \\
\frac{\partial V_{g}}{\partial t} & =V_{g}\left(\frac{1}{p} \frac{\partial p}{\partial t}\right)-\left(V_{a} \alpha_{a}+V_{b} \alpha_{b}\right) \frac{\partial T}{\partial t} .
\end{aligned}
$$

To write mass conservation in a format appropriate for COMSOL, we again need the concentration $c_{b}$ using (27). Similarly, we also define the concentration of anthracite $c_{a}:=\phi_{a} \rho_{a} / M_{a}$ where $M_{a}=0.012 \mathrm{kgmol}^{-1}$ is the molar mass of anthracite. Again neglecting all deformation terms, we arrive at a reduced model comprising (30), (35), (34), plus revised conservation of mass equations:

$$
\begin{aligned}
\frac{\partial c_{a}}{\partial t} & =-c_{a}\left(\frac{1}{V} \frac{\partial V}{\partial t}\right) \\
\frac{\partial c_{b}}{\partial t} & =-I_{v}-c_{b}\left(\frac{1}{V} \frac{\partial V}{\partial t}\right) \\
\frac{\partial\left(\phi_{g} \rho_{g}\right)}{\partial t}+\nabla \cdot\left(\phi_{g} \rho_{g} \mathbf{v}_{g}\right) & =M_{w} I_{v}-\phi_{g} \rho_{g} \frac{1}{V}\left(\frac{\partial V}{\partial t}+\mathbf{v}_{g} \cdot \nabla V\right) .
\end{aligned}
$$

The variables not defined by these differential equations are: $\phi_{a}$ and $\phi_{b}$, calculated from $c_{a}$ and $c_{b}$ as per (27); $\phi_{g}$, calculated as $\left(1-\phi_{a}-\phi_{b}\right)$; and $V, V_{a}$, and $V_{b}$, calculated from $V_{g}$ and $\phi_{k}$ (for each $k$ ). In this model, by allowing the representative volume $V$ to vary, we get a measure of the size of the local deformations, which would provide information about where cracks are most likely to form (i.e. regions where $V$ increases the most). 

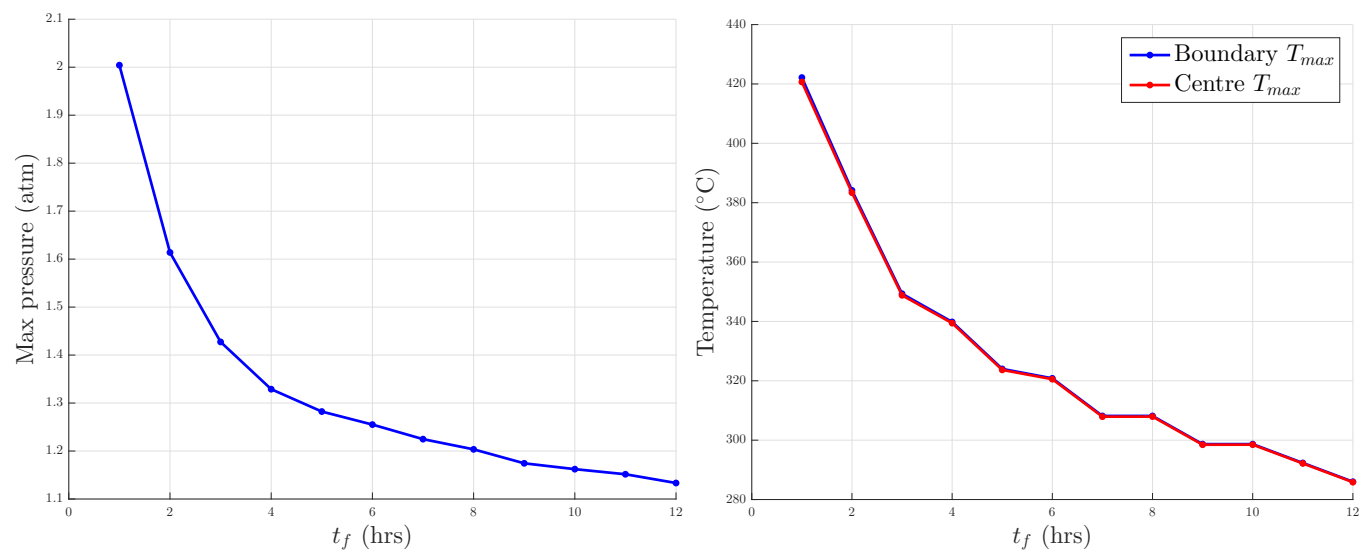

Figure 10: Coal tar pitch model - dependency of maximum pressure and $T_{\max }$ on total baking time $t_{f}$.
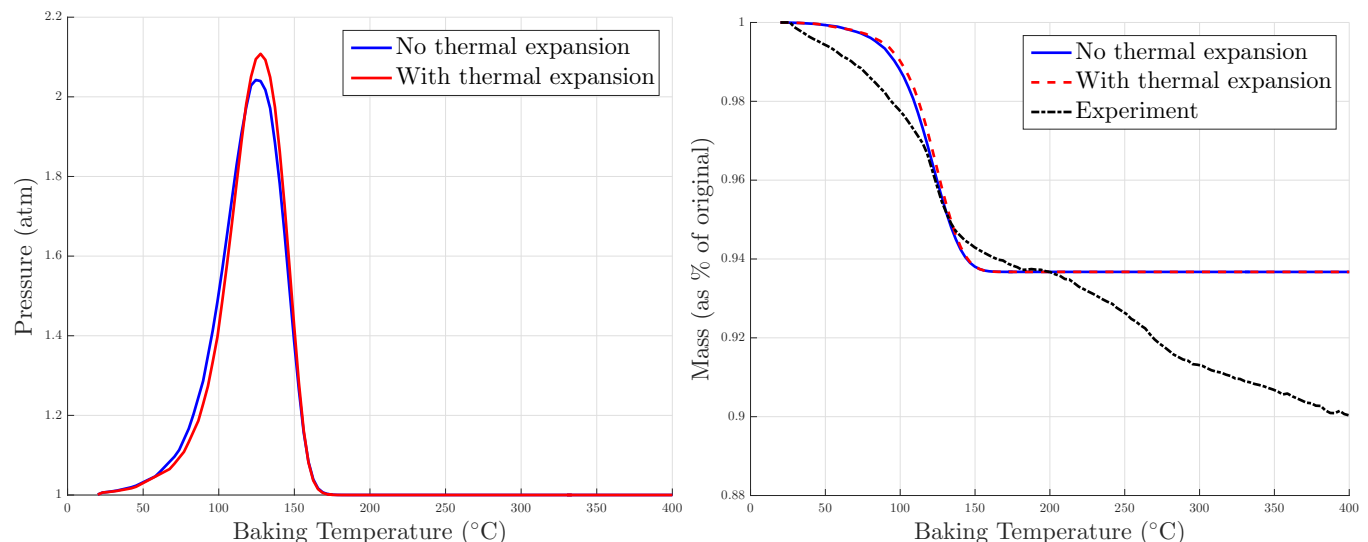

Figure 11: Impact of including thermal expansion of water in the simplified model from Section 3.1.

To give a first indication of how this model would change the results of Section 3.2, we first consider the simplified model from Section 3.1, augmented with thermal expansion of water as per (34). Previously no thermal expansion (i.e. $\alpha_{b}=0$, or equivalently constant $\rho_{b}$ ) was used. These results are shown in Figure 11. We can see that adding thermal expansion of water has a noticeable impact on the maximum pressure achieved, but does not significantly change the mass loss profile.

\section{Conclusions}

Ramming paste is an important product for the metallurgy industry, and Elkem's development of non-toxic binders makes this product safer for workers. A mathematical model of the baking of ramming paste, as presented here, can help to predict the formation of cracks, ultimately improving the performance these binders. In this paper, we have outlined a comprehensive mathematical model of the baking process in Section 2 . This incorporates heat and mass transfer resulting from external heating and evaporation.

In Section 3, we show numerical simulations for a simplified model of heat and mass transfer. This model provides a good fit to experimental data, and provides useful predictions about the effects of varying the baking temperature profile, relative proportions of each component in the paste, and the external pressure. We note that the model results are particularly sensitive to initial porosity, and that the risk of cracking may be reduced by a slower baking rate near $100 \mathrm{C}$ and a lower initial volume fraction of water in the binder. We have also shown how the simplified model may be adapted to ramming paste with a coal tar pitch binder.

There are a number of areas where future work could provide improvements. Firstly, more model validation against experimental results would give greater confidence in the predictions. In particular, we could achieve a greater understanding of what maximum pressures in the simplified model lead to cracking being observed. Incorporating more of the physics from Section 2 would give greater insight into the formation of cracks and changes in porosity. The simple model outlined in Section 3.5 could be a starting point for this work. The model may be extended by considering more realistic evaporation models, e.g. from $[10,19,20]$. The parameters $A_{1}$ and $A_{2}$ may be adjusted to get a good fit with experimental data at given conditions. Our present model also behaves nicely numerically, giving results which show the amount of remaining binder move asymptotically to zero while never going negative. However, the lack of pressure dependency does not lead to plausible responses when the permeability or boundary conditions are changed. Also, our model does not give evaporation from the outer surface if there is no initial porosity $\left(\phi_{g}=0\right)$. Finally, it does not allow condensation, since the resulting value is always positive. While this is not a problem for the current model, as we only model 
heating, if the model is expanded to cooling, condensation will be important. Finally, the addition of more reactions following the approach of [9], or extending the model to include solid mechanics effects such as solid deformations, while using concepts from elastic damage [21, 1] or double porosity models [8, 22], may be beneficial.

\section{Acknowledgments}

This publication is based on work supported by the EPSRC Centre for Doctoral Training in Industrially Focused Mathematical Modelling (EP/L015803/1) in collaboration with Teknova and Elkem. L. Roberts thanks Teknova and Elkem for financial support and the opportunity to work on-site during parts of this project.

\section{References}

[1] F.-J. Ulm, O. Coussy, Z. P. Bažant, The Chunnel Fire. I: Chemoplastic Softening in Rapidly Heated Concrete, J. Eng. Mech. 125 (3) (1999) 272 282. doi:10.1061/(ASCE)0733-9399(1999)125:3(272).

[2] Y. Zhou, R. K. N. D. Rajapakse, J. Graham, A coupled thermoporoelastic model with thermo-osmosis and thermal-filtration, Int. J. Solids Struct. 35 (34-35) (1998) 4659-4683. doi:10.1016/S0020-7683(98)00089-4.

[3] S. S. Sablani, M. Marcotte, O. D. Baik, F. Castaigne, Modeling of simultaneous heat and water transport in the baking process, LWT - Food Sci. Technol. 31 (1998) 201-209. doi:10.1006/fstl.1997.0360.

[4] National Toxicology Program, Coal tars and coal-tar pitches., 13th Rep. Carcinog. 2 (8007) (2014) 111-113.

[5] O. Coussy, Poromechanics, John Wiley \& Sons, Ltd, Chichester, UK, 2004. doi:10.1002/0470092718.

[6] R. Gelet, B. Loret, N. Khalili, A thermo-hydro-mechanical coupled model in local thermal non-equilibrium for fractured HDR reservoir with double porosity, J. Geophys. Res. Solid Earth 117 (7) (2012) 1-23. doi:10.1029/2012JB009161.

[7] B. Loret, N. Khalili, A three-phase model for unsaturated soils, Int. J. Numer. Anal. Methods Geomech. 24 (11) (2000) 893-927. doi:10.1002/1096-9853(200009)24:11;893::AID-NAG105;3.0.CO;2-V.

[8] J. Ma, G. Zhao, N. Khalili, A fully coupled flow deformation model for elasto-plastic damage analysis in saturated fractured porous media, Int. J. Plast. 76 (2016) 29-50. doi:10.1016/j.ijplas.2015.07.011.

[9] M. Jacobsen, M. C. Melaaen, Numerical Simulation of the Baking of Porous Anode Carbon in a Vertical Flue Ring Furnace, Numer. Heat Transf. Part A Appl. 34 (6) (1998) 571-598. doi:10.1080/10407789808914004.

[10] N. T. Fadai, J. Melrose, C. P. Please, A. Schulman, R. A. Van Gorder, A heat and mass transfer study of coffee bean roasting, Int. J. Heat Mass Transf. 104 (2017) 787-799. doi:10.1016/j.ijheatmasstransfer.2016.08.083.

[11] D. A. Nield, A. Bejan, Convection in porous media, 4th Edition, Springer, New York, 2013. doi:10.1007/978-1-4614-5541-7.

[12] Entegris, Properties and Characteristics of Graphite, Tech. rep., Entegris, Inc. (2013).

URL https://www.entegris.com/resources/assets/ 6205-7329-0513.pdf

[13] Engineering Toolbox, Water - Thermal Properties, http://www . engineeringtoolbox.com/water-thermal-properties-d_162. html, accessed: 2016-08-12 (2016).

URL http://www.engineeringtoolbox.com/ water-thermal-properties-d $\left\{\backslash_{-}\right\} 162 . \mathrm{html}$

[14] Engineering Toolbox, Thermal Conductivity of some common Materials and Gases, http://www.engineeringtoolbox.com/ thermal-conductivity-d_429.html, accessed: 2016-08-12 (2016). URL http://www.engineeringtoolbox.com/ thermal-conductivity-d\{\_\}429.html
[15] Engineering Toolbox, Steam Viscosity, http://www. engineeringtoolbox.com/steam-viscosity-d_770.html, accessed: 2016-08-12 (2016).

URL http: //www.engineeringtoolbox.com/ steam-viscosity-d\{\_\}770.html

[16] Engineering Toolbox, Solids - Specific Heats, http://www . engineeringtoolbox.com/specific-heat-solids-d_154.html, accessed: 2016-09-21 (2016).

URL http://www.engineeringtoolbox.com/ specific-heat-solids-d\{\_\}154.html

[17] Engineering Toolbox, Air Properties, http://www. engineeringtoolbox.com/air-properties-d_156.html, accessed: 2016-09-21 (2016).

URL

http://www.engineeringtoolbox.com/ air-properties-d\{\_\}156.html

[18] D. R. Lide, CRC Handbook of Chemistry and Physics, 84th Edition, CRC Press, 2004.

[19] COMSOL Multiphysics, Evaporation in Porous Media with Small Evaporation Rates, Tech. rep. (2016).

URL https://www.comsol.no/model/download/326421/ models.heat.evaporation $\left\{\backslash_{-}\right\}$porous $\left\{\backslash_{-}\right\} \operatorname{media}\left\{\backslash_{-}\right\}$small $\left\{\backslash_{-}\right.$ \}rate.pdf

[20] W. N. Hernández-Díaz, I. I. Ruiz-López, M. A. Salgado-Cervantes, G. C. Rodríguez-Jimenes, M. A. García-Alvarado, Modeling heat and mass transfer during drying of green coffee beans using prolate spheroidal geometry, J. Food Eng. 86 (1) (2008) 1-9. doi:10.1016/j.jfoodeng.2007.08.025.

[21] F.-J. Ulm, O. Coussy, Strength Growth as Chemo-Plastic Hardening in Early Age Concrete, J. Eng. Mech. 122 (12) (1996) 1123-1132. doi:10.1061/(ASCE)0733-9399(1996)122:12(1123).

[22] P. Grassl, M. Jirásek, Damage-plastic model for concrete failure, Int. J. Solids Struct. 43 (22-23) (2006) 7166-7196. doi:10.1016/j.ijsolstr.2006.06.032. 